\title{
Graph attention networks for segment labeling in coronary artery trees
}

Hampe, Nils, Wolterink, Jelmer, Collet, Carlos, Planken, Nils, Išgum, Ivana

Nils Hampe, Jelmer M. Wolterink, Carlos Collet, Nils Planken, Ivana Išgum, "Graph attention networks for segment labeling in coronary artery trees," Proc. SPIE 11596, Medical Imaging 2021: Image Processing, 115961I (15 February 2021); doi: 10.1117/12.2581219

SPIE. Event: SPIE Medical Imaging, 2021, Online Only 


\title{
Graph Attention Networks for Segment Labeling in Coronary Artery Trees
}

\author{
Nils Hampe ${ }^{\mathrm{a}, \mathrm{b}}$, Jelmer M. Wolterink ${ }^{\mathrm{a}, \mathrm{c}}$, Carlos Collet $^{\mathrm{d}}$, Nils Planken ${ }^{\mathrm{e}}$, and Ivana Išgum ${ }^{\mathrm{a}, \mathrm{b}, \mathrm{e}}$ \\ ${ }^{a}$ Department of Biomedical Engineering and Physics, Amsterdam University Medical Centers - \\ location AMC, University of Amsterdam, The Netherlands \\ ${ }^{\mathrm{b}}$ Amsterdam Cardiovascular Sciences, Amsterdam University Medical Center, The Netherlands \\ ${ }^{\mathrm{c}}$ Department of Applied Mathematics, Technical Medical Centre, University of Twente, \\ Enschede, Netherlands \\ ${ }^{\mathrm{d} C a r d i o v a s c u l a r}$ Center Aalst, OLV Clinic, Belgium

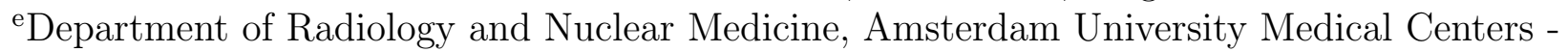 \\ location AMC, The Netherlands
}

\begin{abstract}
Accurately labeled segments of the coronary artery trees are important for diagnostic reporting of coronary artery disease. As current automatic reporting tools do not consider anatomical segment labels, accurate automatic solutions for deriving these labels would be of great value. We propose an automatic method for labeling segments in coronary artery trees represented by centerlines automatically extracted from CCTA images. Using the connectivity between the centerlines, we construct a tree graph. Coronary artery segments are defined as edges of this graph and characterized by location and geometry features. The constructed coronary artery tree is transformed into a linegraph and used as input to a graph attention network, which is trained to classify labels of coronary artery segments. The method was evaluated on 71 CCTA images, achieving an F1-score of $92.4 \%$ averaged over all patients and segments. The results indicate that graph attention networks are suitable for coronary artery tree labeling.
\end{abstract}

Keywords: Artery labeling, graph convolutional networks, graph attention networks, coronary arteries, cardiac CT angiography

\section{INTRODUCTION}

Diagnosis and treatment of coronary artery disease requires reproducible reporting of the presence of atherosclerotic plaque and coronary artery stenosis. The analysis of the coronary arteries typically relies on coronary CT angiography (CCTA) images, in which an intravenously administered contrast agent allows visualization of the morphology of the coronary arteries (Figures 1 and 2). ${ }^{1,2}$ In addition to describing plaque burden and grade of stenosis, clinical reporting of coronary artery disease includes the anatomical label of the coronary artery segment the plaque or stenosis resides in, as defined by the American Heart Association (AHA). ${ }^{3-5}$ Despite recent advances, current automatic approaches typically quantify and characterize plaque without reporting its anatomical location. ${ }^{6}$ Automatic reporting of anatomical segment labels would enhance these tools substantially by establishing a closer link to cardiac pathophysiology.

Previously published methods for automatic labeling of coronary artery segments in CCTA utilized either atlas-based approaches ${ }^{7-9}$ or machine learning. ${ }^{10,11}$ Atlas-based methods perform matching between an unseen tree and a labeled atlas tree. These methods reported good performance but they require careful tuning to deal with substantial anatomical variability. Machine learning-based segment labeling methods have used handcrafted features describing each segment's geometry ${ }^{10,11}$ and location. ${ }^{11}$ Akinyemi et al. ${ }^{10}$ trained a Gaussian classifier to predict labels for each segment individually, agnostic to the information regarding adjacent segments. Hence, subsequent postprocessing exploiting segment connectivity was required. Wu et al. ${ }^{11}$ first leveraged deep learning by training an architecture based on long short-term memory (LSTM) to directly learn multi-segment representations from input trees.

Medical Imaging 2021: Image Processing, edited by Ivana Išgum,

Bennett A. Landman, Proc. of SPIE Vol. 11596, 115961I · @ 2021

SPIE · CCC code: $1605-7422 / 21 / \$ 21 \cdot$ doi: $10.1117 / 12.2581219$

Proc. of SPIE Vol. 11596 115961l-1 

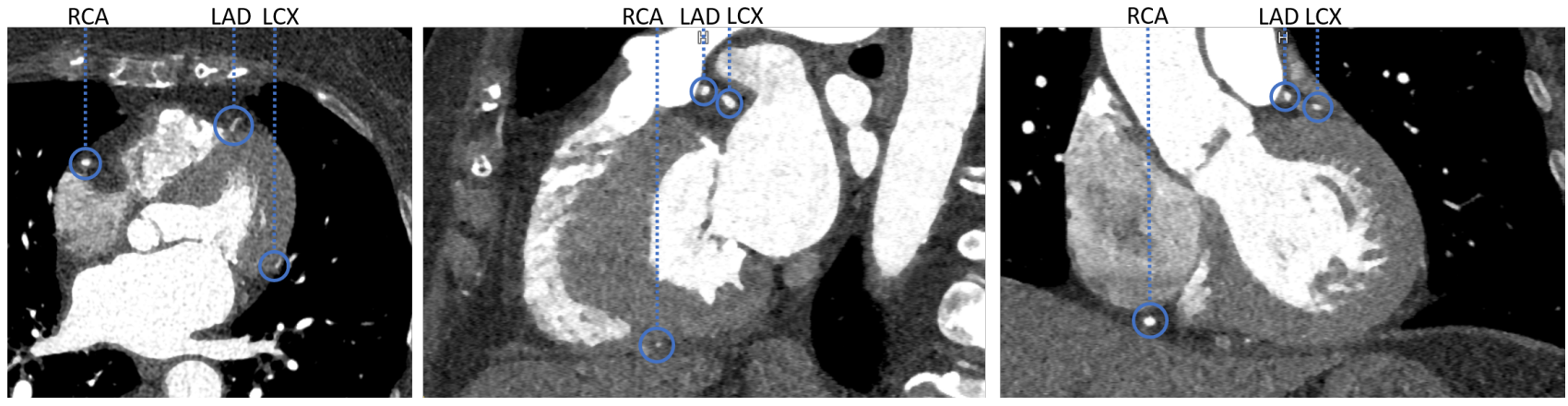

Figure 1: Axial, sagittal and coronal (left to right) slices of a coronary CT angiography image of the heart with labels assigned to the left anterior descending (LAD), left circumflex (LCX), and right coronary artery (RCA).

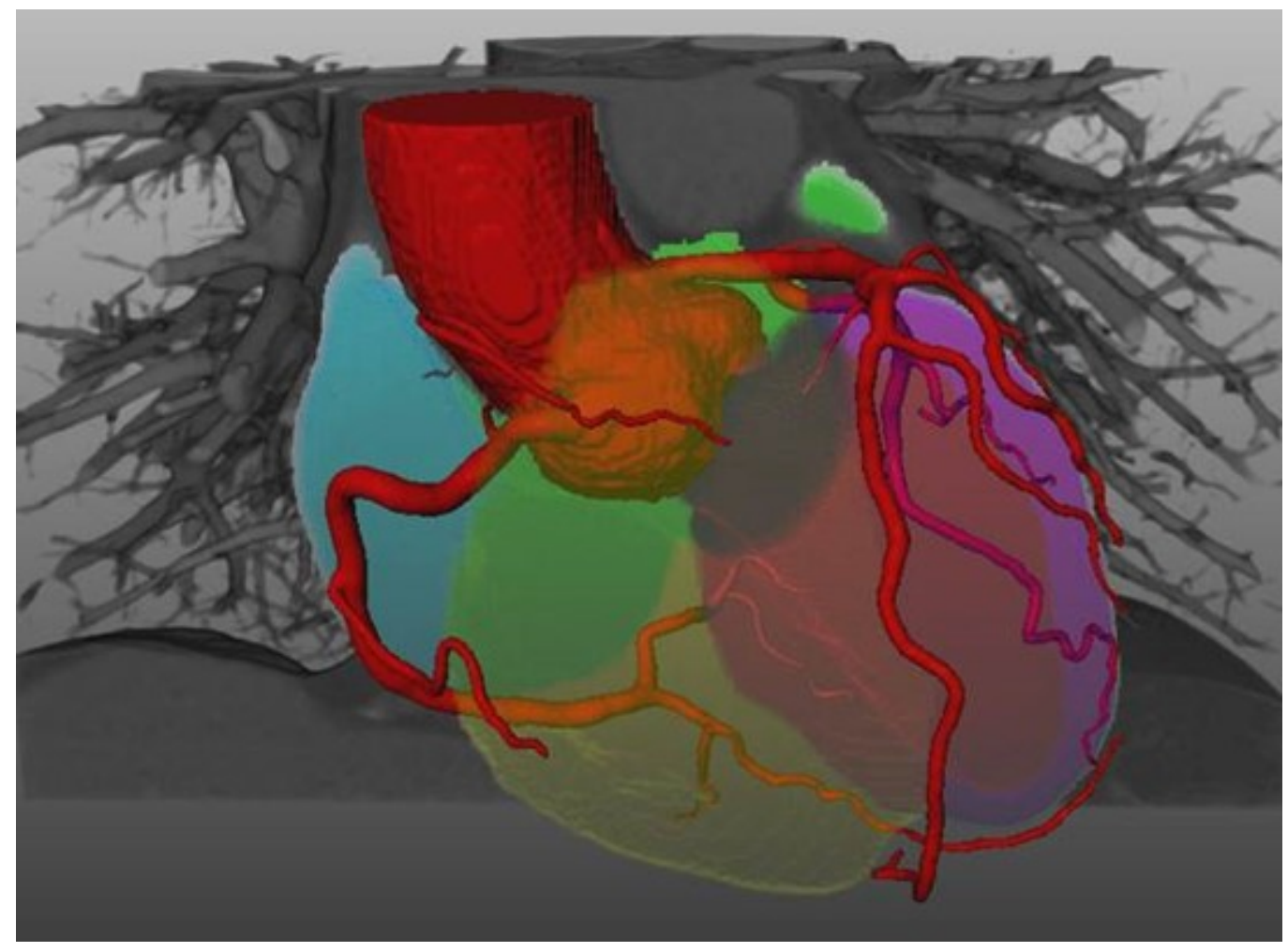

Figure 2: Volume rendering of a coronary CT angiography image of the heart. Highlighted structures are: aorta and coronary artery tree (red), left ventricle (purple), right ventricle (yellow), left atrium (green) and right atrium (blue). 
Here, we present a novel approach to coronary tree segment labeling that represents the coronary tree segments as nodes in a graph and directly performs machine learning on this graph. To this end, we use graph convolutional neural networks (GCNs), which are explicitly designed to model and learn from relationships between nodes in graph structures. We show that graph attention networks (GAT), ${ }^{12}$ a type of GCN, are an effective tool for labeling of coronary artery tree segments.

\section{MATERIALS AND METHODS}

\subsection{CT data}

CCTA images of 71 patients (age range 47-85 years) were used. The scans were acquired between 2016 and 2019 in three different centers: 17 scans from the Tsuchiura Kyodo General Hospital in Tsuchiura (Japan) acquired with a Toshiba Aquilion One, 24 scans from the OLV Ziekenhuis in Aalst (Belgium) acquired with a Siemens Somatom Definition Flash, and 30 scans from the Amsterdam University Medical Centers, location AMC, in Amsterdam (the Netherlands) acquired with a Siemens Somatom Force. Tube voltages between 70 and $120 \mathrm{kVp}$ and tube currents between 66 and $612 \mathrm{mAs}$ were used. All images were reconstructed to an in-plane resolution ranging from 0.29 to $0.49 \mathrm{~mm}^{2}$ with 0.5 to $0.75 \mathrm{~mm}$ slice thickness and increment. Contrast was injected intravenously.

\subsection{Construction of the coronary artery tree}

For each CT image, a graph structure representing the coronary artery tree with segment labels was semiautomatically obtained. Coronary artery centerlines were extracted using our previously developed method described by Wolterink et al., ${ }^{13}$ employing a convolutional neural network to track each artery from the ostium to its most distal part based on a single seed point. Seed points were automatically identified and - where necessary - supplemented with manually placed seed points to ensure completeness of the coronary artery trees. Subjects were only included in this study if all visible centerlines could be successfully extracted. For every patient, this resulted in a set of centerlines, each consisting of a list of centerline points.

Centerlines were transformed into a graph structure as follows. One of the extracted centerlines was randomly selected as the initial tree. Each of the remaining centerlines was then added to the tree by determining the overlap with the existing tree and appending the non-overlapping part. As all centerlines reach one ostium, overlap of new segments with the already existing tree was removed. In the process, connectivity between centerlines was recorded through a tree graph $\hat{\mathcal{G}}=(\hat{\mathcal{V}}, \hat{\mathcal{E}})$, where the nodes $\hat{\mathcal{V}}$ were represented by ostia (roots)/bifurcations/distal points (leafs); and where the edges $\hat{\mathcal{E}}$ corresponded to coronary artery tree segments (Figure 3). The tree graph $\hat{\mathcal{G}}$ was postprocessed by removing leaf segments with lengths shorter than $5 \mathrm{~mm}$, as these were likely noise from repetitive tracking of the same branch. To adapt to the node classification schemes of typical GCN implementations we transformed $\hat{\mathcal{G}}$ into a so-called linegraph $\mathcal{G}=(\mathcal{V}, \mathcal{E})$, in which original edges $\hat{\mathcal{E}}$ became the new nodes $\mathcal{V}$ (Figure 4). Edges connected by a node in $\hat{\mathcal{G}}$ were pairwise connected by edges in $\mathcal{G}$.

\subsection{Reference standard}

To define the reference standard, anatomical segment labels were assigned to the nodes in $\mathcal{G}$ according to the model introduced by the AHA. The following labels were assigned: left main (LM), left anterior descending (LAD), left circumflex (LCX), right coronary artery (RCA), diagonal (D), septal (S), obtuse marginal (OM), acute marginal (AM), right posterior descending artery (R-PDA), right posterolateral branch (R-PLB). Due to the relatively low number of samples of the segments predominantly occurring in the rare left dominant systems, the left posterolateral segments were labeled as OM, and the left posterior descending arteries were labeled as LCX. Furthermore, defining segments as branches between bifurcations prevented accurate sub-divisions of the main arteries. Therefore, in line with other data-driven methods ${ }^{10,11}$ a single label was utilized for each of the three main arteries. Figure 5 shows reference labels in two semi-automatically extracted coronary artery trees. 


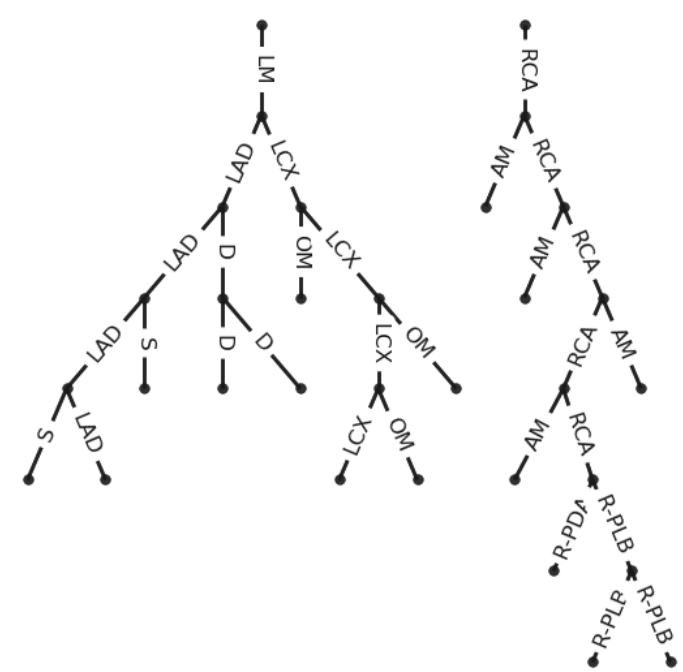

Figure 3: Tree graph $\hat{\mathcal{G}}$ with roots/bifurcations/leafs as nodes $\hat{\mathcal{V}}$, and segments as edges $\hat{\mathcal{E}}$. The following labels were assigned: left main (LM), left anterior descending (LAD), left circumflex (LCX), right coronary artery (RCA), diagonal (D), septal (S), obtuse marginal $(\mathrm{OM})$, acute marginal $(\mathrm{AM})$, right posterior descending artery (R-PDA), right posterolateral branch (R-PLB).
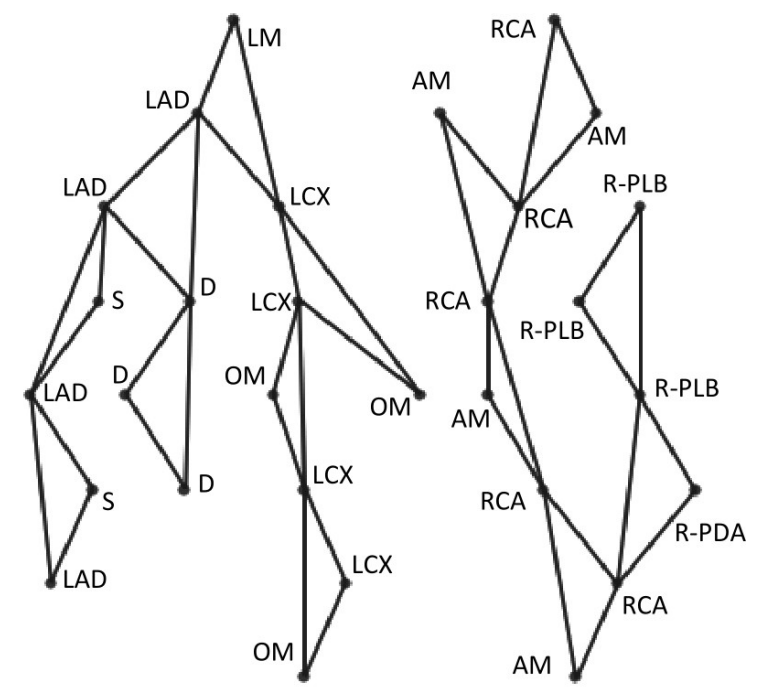

Figure 4: Linegraph $\mathcal{G}$, with segments as nodes $\mathcal{V}$. The following labels were assigned: left main (LM), left anterior descending (LAD), left circumflex (LCX), right coronary artery (RCA), diagonal (D), septal (S), obtuse marginal $(\mathrm{OM})$, acute marginal $(\mathrm{AM})$, right posterior descending artery (R-PDA), right posterolateral branch (R-PLB).

\subsection{Automatic labeling of coronary artery segments}

We develop a GCN to classify the nodes $\mathcal{V}$ according to the corresponding anatomical segments. GCNs are a generalization of convolutional neural networks $(\mathrm{CNNs})$ to arbitrary graph inputs. A graph $\mathcal{G}$ is represented by a set of nodes $\mathcal{V}$, each described by a feature vector $\vec{h}$, which are connected by edges $\mathcal{E}$. Therefore, in GCNs, filter kernels of CNNs are replaced by a permutation invariant aggregation function that combines features of connected nodes. The GCN employed in this work is a GAT. ${ }^{12}$ A GAT aggregates features $\overrightarrow{h_{j}}$ of connected nodes $\mathcal{N}_{i}$, transformed by weight matrix $\mathbf{W}$, using a weighted average $\vec{h}_{i}{ }^{\prime}=\sigma\left(\sum_{j \in \mathcal{N}_{i}} \alpha_{i j} \mathbf{W} \vec{h}_{j}\right)$, with $\sigma$ as the LeakyReLU nonlinearity. Weighting coefficients $\alpha_{i j}$ are determined by attention subnetworks (heads), parameterized by weight vectors $\overrightarrow{\mathbf{a}}$, as

$$
\alpha_{i j}=\frac{\exp \left(\sigma\left(\overrightarrow{\mathbf{a}}^{T}\left[\mathbf{W} \overrightarrow{h_{i}} \| \mathbf{W} \overrightarrow{h_{j}}\right]\right)\right)}{\sum_{k \in \mathcal{N}_{i}} \exp \left(\sigma\left(\overrightarrow{\mathbf{a}}^{T}\left[\mathbf{W} \overrightarrow{h_{i}} \| \mathbf{W} \overrightarrow{h_{k}}\right]\right)\right)},
$$

where || denotes concatenation.

In this work, the original GAT implementation is augmented by introducing dense connections from the input features to all consecutive GAT-layers. Three GAT layers are used with four heads per layer, eight encodings per head, and residual connections. Finally, a softmax layer predicts output probabilities for 10 classes corresponding to the above described segment labels.

\subsubsection{Node features}

We make use of location features describing position and orientation of each segment, transformed into a relative coordinate system. As origin we utilize the center of mass of the left ventricle myocardium, which we segment using a fully convolutional residual network. ${ }^{14}$ 

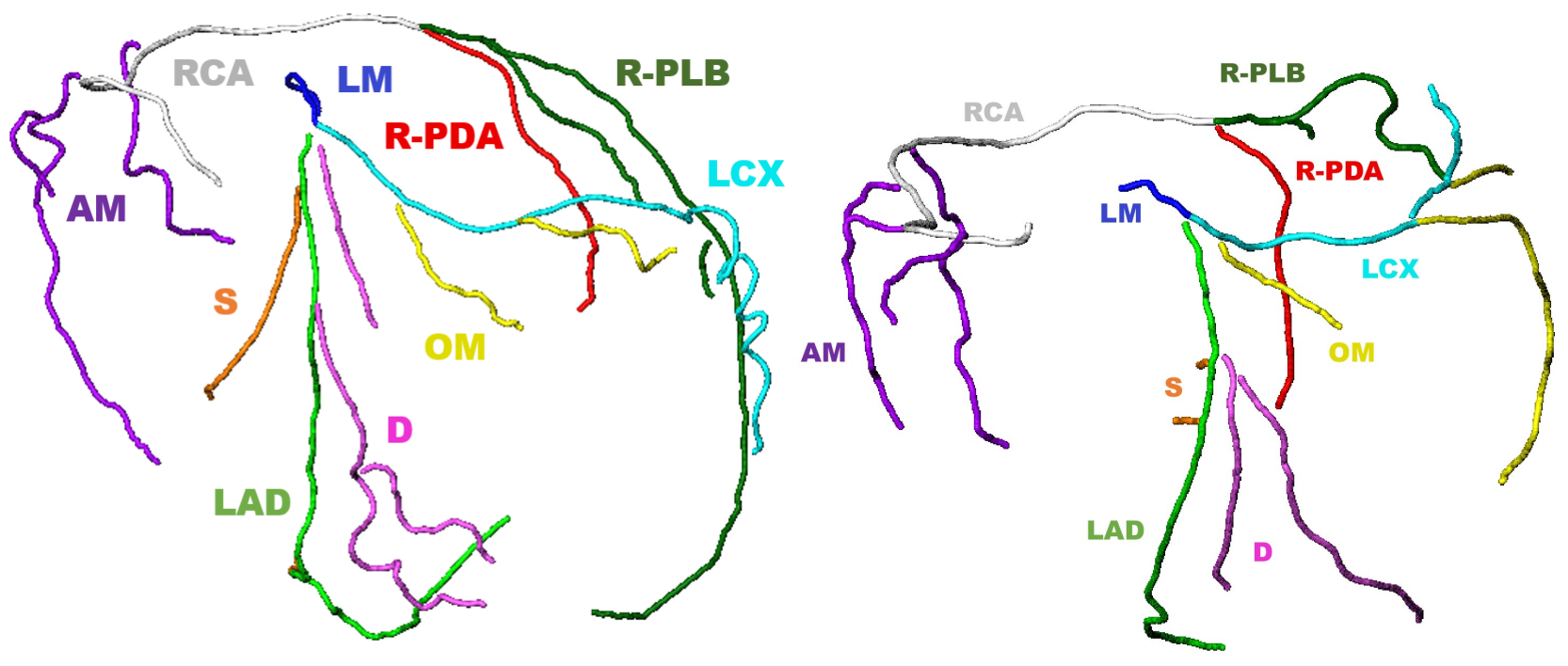

Figure 5: Labeled coronary artery trees from the reference standard. The following labels were assigned: left main (LM), left anterior descending (LAD), left circumflex (LCX), right coronary artery (RCA), diagonal (D), septal (S), obtuse marginal (OM), acute marginal (AM), right posterior descending artery (R-PDA), right posterolateral branch (R-PLB).

For each segment we extract Cartesian coordinates $x, y$ and $z$ from points located at $0 \%, 25 \%, 50 \%, 75 \%$, $100 \%$ of the centerline (15 features). Additionally, the radius outputs of the centerline tracker are refined by calculating mean and standard deviation over all radii in a segment (2 features). Information about tree direction is injected by 3 additional order features, describing depth of the segment in the tree as well as maximum and relative depth of the sub-tree downstream to the segment. All features are scaled to the same order of magnitude.

\section{EXPERIMENTS AND RESULTS}

To evaluate the method, we perform ten-fold cross validation with datasets from all three hospitals distributed evenly across folds. All networks are trained for 4000 epochs with a batch size of 1, where an epoch corresponds to processing every sample in the training set exactly once. We use the ADAM optimizer with a learning rate of 0.001 and cross-entropy loss.

Table 1 lists results of the proposed method, compared with previous works. Note that each method evaluated performance using a different set of CCTA images. Hence, direct comparison can not be made and presented results need to be interpreted with caution. The table shows that our results for the main arteries (LM, LAD, LCX, RCA) are comparable with the results of the previously reported methods. Unlike other data-driven approaches, our method achieved high precision and recall for the right posterior descending artery (R-PDA) and for the right posterolateral branch (R-PLB). Moreover, the here proposed method outperforms all previous methods for septal branches (S). The presented approach achieved an F1-score of $92.4 \%$ for all classes combined.

Several experiments were performed to evaluate our methodological choices. To test the necessity of dense connections to the input features, we evaluated the combined performance of a GAT without dense connections (GAT-ND), which yielded an F1-score of $85.4 \%$. Furthermore, utility of information about connectivity is investigated through two ablation experiments where we trained a GAT with one layer instead of three (GATL1), and a standalone multi layer perceptron (MLP) with two layers and eight neurons in the hidden layer. Evaluations yielded average F1-scores of $87.5 \%$ for GAT-L1 and $85.5 \%$ for MLP. 
Table 1: Precision and recall for the proposed and previously reported methods. Note that the majority of studies only presented one of these metrics. A single number for a segment with multiple sub-segments represents an average over all sub-segments.

\begin{tabular}{|c|c|c|c|c|c|c|c|c|c|c|c|c|c|c|c|c|c|c|c|}
\hline & & LM & & LCX & & & LAD & & & $\mathrm{RCA}$ & & R-PDA & R-PLB & 1 & & $\mathrm{AM}$ & $\mathrm{O}$ & & $\mathrm{S}$ \\
\hline sub-segment & & & $\mathrm{p}$ & $\mathrm{m}$ & d & $\mathrm{p}$ & $\mathrm{m}$ & d & $\mathrm{p}$ & $\mathrm{m}$ & d & & & D1 & D2 & & OM1 & OM2 & \\
\hline $\begin{array}{l}\text { Yang et al. }{ }^{7} \\
\text { Gülsün et al. }{ }^{8} \\
\text { Cao et al. }{ }^{9} \\
\text { Wu et al. }{ }^{11} \\
\text { proposed }\end{array}$ & 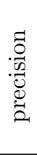 & $\begin{array}{r}100 \\
100 \\
100 \\
99.1 \\
92.2\end{array}$ & $\begin{array}{r}84.5 \\
100 \\
87.2 \\
93.5 \\
92.3\end{array}$ & $\begin{array}{r}80.5 \\
100 \\
81.7\end{array}$ & 84.0 & $\begin{array}{r}94.0 \\
100 \\
94.0 \\
96.9 \\
91.8\end{array}$ & $\begin{array}{l}86.5 \\
97.5 \\
88.5\end{array}$ & $\begin{array}{r}94.0 \\
100 \\
95.7\end{array}$ & $\begin{array}{r}97.5 \\
100 \\
99.3 \\
96.0 \\
91.9\end{array}$ & $\begin{array}{r}96.0 \\
100 \\
97.3\end{array}$ & $\begin{array}{r}92.5 \\
100 \\
92.5\end{array}$ & $\begin{array}{l}96.5 \\
95.5 \\
96.5 \\
79.8 \\
91.9\end{array}$ & $\begin{array}{l}89.5 \\
91.0 \\
89.0 \\
82.7 \\
89.2\end{array}$ & $\begin{array}{l}99.0 \\
91.0 \\
93.7 \\
91.0 \\
96.1\end{array}$ & $\begin{array}{l}86.5 \\
83.0 \\
82.0\end{array}$ & 98.6 & $\begin{array}{l}85.0 \\
90.0 \\
90.1 \\
85.2 \\
87.1\end{array}$ & $\begin{array}{l}79.5 \\
83.0 \\
79.0\end{array}$ & $\begin{array}{l}76.0 \\
75.9 \\
90.6\end{array}$ \\
\hline $\begin{array}{l}\text { Akinyemi et al. }{ }^{10} \\
\text { Wu et al. } \\
\text { proposed }\end{array}$ & 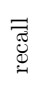 & $\begin{array}{r}100 \\
98.6 \\
97.1\end{array}$ & $\begin{array}{l}91.7 \\
90.0 \\
84.1\end{array}$ & & & $\begin{array}{l}97.4 \\
97.1 \\
97.8\end{array}$ & & & $\begin{array}{l}98.9 \\
94.2 \\
97.6\end{array}$ & & & $\begin{array}{l}65.0 \\
76.2 \\
84.4\end{array}$ & $\begin{array}{l}86.5 \\
90.6 \\
91.3\end{array}$ & $\begin{array}{l}80.0 \\
96.5 \\
88.3\end{array}$ & & 96.3 & $\begin{array}{l}78.9 \\
90.5 \\
91.3\end{array}$ & & $\begin{array}{l}89.2 \\
91.9\end{array}$ \\
\hline
\end{tabular}

\section{DISCUSSION AND CONCLUSION}

In the presented approach, input features were concatenated to each consecutive layer input, which, when omitted, decreased performance by $7 \%$. This indicates importance of these features, which is in line with relatively good F1-score (85.5\%) of the MLP. The reason for equal performance of GAT-ND and MLP could be that the aggregation operations in the GAT layers discard important information from input features. Nevertheless, we demonstrated that information from surrounding segments, encoded by the GAT layers, increases performance of automatic coronary artery tree labeling. Increasing the number of layers from one to three yielded a performance gain of almost $5 \%$, demonstrating utility of information from the local environment.

Deep learning algorithms typically require large amounts of data for good performance. For example, the deep learning algorithm proposed by Wu et al. ${ }^{11}$ used 392 subjects in training. However, the presented results demonstrate that highly accurate coronary artery tree labeling can be performed using a GAT trained with as few as 64 subjects. Future work will evaluate potential performance gains with a larger training dataset, which can potentially be enhanced by augmentation through discarding random parts of the trees.

Moreover, the limited set of training data resulted in very few samples of branches only present in left dominant systems. Hence, we combined these segments with other classes. A larger dataset with more examples of rarely occurring segments may enable predicting these classes as well. More data might also enable training a deeper network with larger receptive field. This could enable employing a more fine-grained segment definition which could be used to separately predict sub-segments of the main arteries, i.e. proximal, mid and distal parts.

To conclude, we presented a deep learning approach for labeling of the coronary artery segments in CCTA exams. For this purpose, we described methods for extracting graphs and features from CCTA images. The results show similar performance to previous approaches for most branches, and improved performance for the small leaf branches S and R-PLB, despite the limited dataset size used in training.

\section{Acknowledgement}

This work has been financially supported by PIE Medical Imaging B.V..

\section{REFERENCES}

[1] Zreik, M., van Hamersvelt, R. W., Wolterink, J. M., Leiner, T., Viergever, M. A., and Isgum, I., "A Recurrent CNN for Automatic Detection and Classification of Coronary Artery Plaque and Stenosis in Coronary CT Angiography," IEEE Trans Med Imaging 38(7), 1588-1598 (2019).

[2] Chen, M., Wang, X., Hao, G., Cheng, X., Ma, C., Guo, N., Hu, S., Tao, Q., Yao, F., and Hu, C., "Diagnostic performance of deep learning-based vascular extraction and stenosis detection technique for coronary artery disease," Brit J Radiol 93, 20191028 (Mar. 2020). Publisher: The British Institute of Radiology. 
[3] Austen, W. G., Edwards, J. E., Frye, R. L., Gensini, G. G., Gott, V. L., Griffith, L. S., McGoon, D. C., Murphy, M. L., and Roe, B. B., "A reporting system on patients evaluated for coronary artery disease. Report of the Ad Hoc Committee for Grading of Coronary Artery Disease, Council on Cardiovascular Surgery, American Heart Association," Circulation 51(4), 5-40 (1975). Publisher: American Heart Association.

[4] Litjens, G., Ciompi, F., Wolterink, J. M., de Vos, B. D., Leiner, T., Teuwen, J., and Išgum, I., "State-of-theArt Deep Learning in Cardiovascular Image Analysis," JACC Cardiovasc Imaging 12(8 Pt 1), 1549-1565 (2019).

[5] Lin, A., Kolossváry, M., Išgum, I., Maurovich-Horvat, P., Slomka, P. J., and Dey, D., "Artificial intelligence: improving the efficiency of cardiovascular imaging," Expert Rev Med Devices 17, 565-577 (June 2020).

[6] Hampe, N., Wolterink, J. M., van Velzen, S. G. M., Leiner, T., and Išgum, I., "Machine Learning for Assessment of Coronary Artery Disease in Cardiac CT: A Survey," Front Cardiovasc Med 6 (2019).

[7] Yang, G., Broersen, A., Petr, R., Kitslaar, P., de Graaf, M. A., Bax, J. J., Reiber, J. H., and Dijkstra, J., "Automatic coronary artery tree labeling in coronary computed tomographic angiography datasets," in [Comput Cardiol], 109-112, IEEE (2011).

[8] Gülsün, M. A., Funka-Lea, G., Zheng, Y., and Eckert, M., "CTA coronary labeling through efficient geodesics between trees using anatomy priors," in $[M I C C A I], \mathbf{1 7}(\mathrm{Pt} 2), 521-528$, The MICCAI Society (2014).

[9] Cao, Q., Broersen, A., de Graaf, M. A., Kitslaar, P. H., Yang, G., Scholte, A. J., Lelieveldt, B. P. F., Reiber, J. H. C., and Dijkstra, J., "Automatic identification of coronary tree anatomy in coronary computed tomography angiography," Int J Cardiovasc Imaging 33(11), 1809-1819 (2017).

[10] Akinyemi, A., Murphy, S., Poole, I., and Roberts, C., "Automatic labelling of coronary arteries," in [Proc Eur Signal Process Conf EUSIPCO], 17, 1562-1566, IEEE (2009).

[11] Wu, D., Wang, X., Bai, J., Xu, X., Ouyang, B., Li, Y., Zhang, H., Song, Q., Cao, K., and Yin, Y., "Automated anatomical labeling of coronary arteries via bidirectional tree LSTMs," Int $J$ Comput Assist Radiol Surg 14(2), 271-280 (2019).

[12] Veličković, P., Cucurull, G., Casanova, A., Romero, A., Liò, P., and Bengio, Y., "Graph Attention Networks," in $[I C L R], 6$ (2018).

[13] Wolterink, J. M., van Hamersvelt, R. W., Viergever, M. A., Leiner, T., and Išgum, I., "Coronary artery centerline extraction in cardiac CT angiography using a CNN-based orientation classifier," Med Image Anal 51, 46-60 (2019).

[14] Bruns, S., Wolterink, J. M., Takx, R. A. P., Hamersvelt, R. W. v., Suchá, D., Viergever, M. A., Leiner, T., and Išgum, I., "Deep learning from dual-energy information for whole-heart segmentation in dual-energy and single-energy non-contrast-enhanced cardiac CT," Med Phys 47(10), 5048-5060 (2020). 